
\title{
25 Research Square \\ Liver Transplantation, IBD Clinical Course, Is There a Link? A Systematic Review and Meta-Analysis Protocol
}

\section{GholamReza Sivandzadeh}

Shiraz University of Medical Sciences

Manoosh Mehrabi

Shiraz University of Medical Sciences

Ali Reza Safarpour ( $\nabla$ safarpourar@gmail.com )

Shiraz University of Medical Sciences https://orcid.org/0000-0002-9880-0043

\section{Hadis Ashrafizadeh}

Ahvaz Jundishapur University of Medical Sciences: Ahvaz Jondishapour University of Medical Sciences

\section{Abbas Ali Keshtkar}

Tehran University of Medical Sciences

\section{Protocol}

Keywords: Liver Transplantation, IBD Clinical Course, Primary Sclerosing Cholangitis (PSC), Crohn's Disease, Ulcerative Colitis

Posted Date: October 19th, 2020

DOl: https://doi.org/10.21203/rs.3.rs-93114/v1

License: (1) This work is licensed under a Creative Commons Attribution 4.0 International License. Read Full License 


\section{Abstract}

\section{Background}

Primary sclerosing cholangitis (PSC) is an uncommon chronic and progressive cholestatic liver disease. There is a robust association between PSC and Inflammatory Bowel Disease (IBD), usually Ulcerative Colitis (UC). According to the review of literature, the incidence of de novo IBD after solid organ transplantation (SOT) is found to be higher than general population. Considering lacking of any systematic review and pursuing debate on the clinical course and risk factors of IBD activity after Liver Transplantation (LT), the present study will be performed with a focus investigation on the correlation of IBD clinical course with liver transplantation.

\section{Methods}

In this systematic review, the electronic databases including PubMed/MEDLINE, Scopus, WoS (Clarivate Analytics), Embase (Embase.com), and ProQuest will be searched. Our search strategy (i.e. The eligibility criteria) covers prospective and retrospective observational studies that evaluated the clinical course of ulcerative colitis or/and Crohn's disease after liver transplantation with no language limitation published between 1970.01.01 and 2020.03.30. The selection phase, data extraction and quality assessment will be independently implemented by two authors. In case of any disagreement between the authors, the issue will be resolved by consensus; if not resolved, the opinion of a third expert will be asked. We will use one of the following two models: Random Effect Model or Fixed Effect Model according to the severity of methodological heterogeneity and forest plot will present the combination of data obtained from all finally included studies, to show the separated and combined frequency and their corresponding $95 \% \mathrm{Cls}$. Statistical heterogeneity will be evaluated by the Q-statistic test and $\mathrm{I}^{2}$ statistics. Funnel plot for assessing the potential reporting bias, Begg's and Egger's tests for meaningful results of the publication bias, and the Fill \& Trim method for corrected publication bias will be used.

\section{Discussion}

This systematic review and meta-analysis study will clarify the correlation of IBD clinical course with liver transplantation. Because of the importance of inflammatory bowel disease, if the future study reveals consistent results, it will be clinically beneficial for physicians and other health care professionals to better manage inflammatory bowel disease after liver transplantation.

Systematic review registration

PROSPERO, CRD42020179412.

\section{Background}

Primary sclerosing cholangitis (PSC), with ICD-10 diagnostic code K83.01, is an uncommon chronic and progressive cholestatic liver disease. It is characterized by fibro inflammatory reactions in the medium to 
large bile ducts. Either intrahepatic or extra hepatic biliary systems could be affected in this disease. Its incidence and prevalence in western countries are reported about 0.07 to 1.3 per $10^{5}$ /person-years and 8.5 to 13.6 per $10^{5}$ populations, respectively. Inflammatory bowel diseases (IBDs), comprising Crohn's disease (CD), with ICD-10 diagnostic code K50.0-50.9 and ulcerative colitis (UC), with ICD-10 diagnostic code $\mathrm{K} 51.0-51.9$, are idiopathic chronic inflammation and ulceration involving mainly the gastrointestinal tract. There is a robust association between PSC and IBD, usually UC. About $50 \%$ to $99 \%$ of patients with PSC are found to have concomitant IBD (mostly UC) across varied studies (1-5).

Primary sclerosing cholangitis generally progresses to biliary cirrhosis and liver failure, often in an insidious manner. The median time from diagnosis to death or liver transplantation (LT) lasts approximately 9 to 18 years in centers with LT facility. Liver transplantation is yet considered to be the only curative treatment for patients with PSC and end -stage liver disease. Pan colitis, right-sided colitis, rectal sparing, and backwash ileitis are more frequently seen in patients with PSC-UC. However, UC usually runs a mild course in patients with concurrent PSC (6-8).

IBD runs a paradoxical course after LT despite potent immunosuppressive agents used for prevention of organ rejection. Both de novo IBD and exacerbation of pre-existing IBD have been reported in the literature. The incidence of de novo IBD after LT is found to be higher than general population reaching $0.2 \%$ per year (9). In one study, 18 patients with UC-PSC were followed before and after LT. The study showed that half of cases had worse UC course after LT and the rest half remained in remission (10). In a similar study, Gelley F et al, applied Mayo score to assess the activity of UC before and after LT in 31 cases with PSC-UC. They found that despite inactive state in $95 \%$ of patients before LT, $40 \%$ of patients experienced severe disease after LT and just 10\% remained inactive (11). Nonetheless, other studies concluded that IBD would remain stable in activity in the majority of PSC-UC cases after LT. In the study performed in 2017, we evaluated 159 patients with PSC-UC before and after LT. Our study demonstrated that no change or even alleviation in IBD symptoms in approximately $94 \%$ of cases after LT and just $6 \%$ experienced symptom worsening (3). Similarly, Navaneethan $\mathrm{U}$ et al, showed that the majority of PSC-UC cases (83\%) had quiescent course after LT and the remainder had exacerbations in symptoms leading to colectomy (12). Several studies remarked possible risk factors of IBD activity after LT embarking tacrolimus, duration after $L T$, and cytomegalovirus $(C M V)$ infection $(3,13,14)$. Considering the lack of any systematic review and pursuing debate on the clinical course and risk factors of IBD activity after LT we decided to conduct a systematic review based on available studies.

\section{Objectives}

\section{Primary Objective}

The primary outcome of this systematic review includes the assessment of the frequency (\%) of IBD patients with exacerbation after LT and the frequency of IBD patients with quiescent underlying IBD after LT.

\section{Secondary Objectives}


1. Estimating the frequency (\%) of patients of $C D, U C$ or both with exacerbation or remission after liver transplantation by age group.

2. Estimating the frequency of patients of $C D, U C$ or both with exacerbation or remission after liver transplantation by gender.

3. Estimating the frequency of patients of $C D, U C$ or both with exacerbation or remission after liver transplantation according to the severity of disease before transplantation.

4. Estimating the frequency of patients of $C D, U C$ or both with exacerbation or remission after liver transplantation according to duration of follow up after liver transplantation.

5. Estimating the frequency of patients of $C D, U C$ or both with exacerbation or remission after liver transplantation according to smokers/ non-smokers patients.

6. Estimating the frequency (\%) of patients with $\mathrm{CD}$ or UC with exacerbation or remission after liver transplantation according to immunosuppressive agents' regimen after LT.

7. Assessing potential heterogeneity in the frequency (\%) of patients of $C D, U C$ or both with exacerbation or remission after liver transplantation and finding its potential causes.

\section{Methods}

This protocol will be reported according to meta-analysis of observational studies in epidemiology guidelines (MOOSE) and preferred reporting items for systematic review and meta-analysis protocols (PRISMA-P) 2015 (15).

\section{Eligibility Criteria of Primary Studies}

\section{Inclusion and exclusion criteria}

\section{Study type}

This systematic review will include prospective and retrospective observational studies that they evaluate the clinical course of ulcerative colitis or/and Crohn's disease after liver transplantation. No restriction on sample size and language will be applied for primary studies to be included.

\section{Type of participants}

All patients with ulcerative colitis or/and Crohn's disease with any age group, including Adult or pediatrics and either gender, who underwent liver transplantation during the course of their disease, will be included in this study.

\section{Search strategy and literature sources}

\section{Search strategy components}


We will search PubMed/ MEDLINE, Scopus, WoS (Clarivate Analytics), Embase (Embase.com), from 01.01.1970 to 30.03.2020. In order to extract the near all relevant studies, search component include the diseases (Inflammatory Bowel Disease, Ulcerative Colitis, Crohn's Disease, Primary Sclerosing Cholangitis and their synonyms) and the intervention (Liver Transplantation), as shown in Table1.

To find the synonyms of search components, thesaurus systems, including Emtree and MeSH, the free text method, the views of experts and also related articles and abstracts will be used.

The other methods used to find relevant studies are manually search in Grey literature (thesis, conference papers, and organizational reports), and contacting the experts to find relevant their unpublished studies and introducing related conferences.

The results of all searches carried out in the various databases and other resources will be collected in Endnote software. 


\section{Table 1 The search strategy used in PubMed/MEDLINE from 01.01.1970 to 30.03.2020}

\section{Number Search terms}

1 (“"Idiopathic Proctocolitis”[tiab] OR “Ulcerative Colitis”[tiab] OR “Colitis Gravis”[tiab] OR ("Inflammatory Bowel Disease" [tiab] AND "Ulcerative Colitis Type"[tiab])OR"chronic ulcerative colitis" [tiab] OR "colitis ulcerative"[tiab] OR "colitis ulcerosa" [tiab] OR "colitis ulcerosa chronic"[tiab] OR (colitis[tiab] AND ulcerative[tiab]) OR (Colitis[tiab] AND mucosal[tiab]) OR (colitis[tiab] AND ulcerous[tiab]) OR (Colon[tiab] AND "chronic ulceration"[tiab]) OR "histiocytic ulcerative colitis"[tiab] OR "mucosal colitis"[tiab] OR "ulcerative colorectitis"[tiab] OR "ulcerative procto colitis"[tiab] OR "ulcerative proctocolitis"[tiab] OR "ulcerous colitis"[tiab])

2 ("Crohn's Enteritis"[tiab] OR "Regional Enteritis"[tiab] OR "Crohn's Disease"[tiab] OR "Crohns Disease"[tiab] OR "Inflammatory Bowel Disease"[tiab] OR “Granulomatous Enteritis"[tiab] OR Ileocolitis[tiab] OR “Granulomatous Colitis"[tiab] OR "Terminal Ileitis"[tiab] OR "Regional Ileitides"[tiab] OR "Regional Ileitis" [tiab] OR "cleron disease"[tiab] OR "Crohn's disease"[tiab] OR "Crohns disease"[tiab] OR "enteritis regionalis"[tiab] OR ("intestinal tract"[tiab] AND "regional enteritis"[tiab]) OR "morbuscrohn"[tiab] OR "regional enterocolitis"[tiab])

3 ("Inflammatory Bowel Disease"[tiab] OR ("Bowel Diseases"[tiab] AND Inflammatory[tiab]) OR "Indeterminate colitis"[tiab] OR “undetermined colitis"[tiab])

4 (Cholangitides[tiab] AND Sclerosing[tiab]) OR "SclerosingCholangitides"[tiab] OR "Sclerosing Cholangitis"[tiab] OR (Cholangiitis[tiab] AND Sclerosing[tiab]) OR (Cholangiitides[tiab] AND Sclerosing[tiab])OR"SclerosingCholangiitides" [tiab]OR "SclerosingCholangiitis"[tiab] OR "Primary Sclerosing Cholangitis"[tiab] OR (Cholangitides[tiab] AND "Primary Sclerosing"[tiab]) OR "Primary SclerosingCholangitides"[tiab] OR "Primary SclerosingCholangitides"[tiab] OR ("Sclerosing Cholangitis"[tiab] AND Primary[tiab]) OR (Cholangitis[tiab] AND "Primary Sclerosing"[tiab])

$5 \quad 1$ OR 2 OR 3 OR 4

6

(liver[tiab] AND transplantation[tiab])OR “Liver Transplantations"[tiab])

7 1990/01/01:2019/12/31[dp]

8 5 AND 6 AND 7

\section{Screening and Selection}

After the searching process and in the screening stage, two authors (ARS and MM) will review the title and abstract of the studies according to the inclusion and exclusion criteria and will find and extract the relevant studies. The studies with insufficient data in one or more aspects of the inclusion criteria will be excluded and a final decision will be made according to their full text in the subsequent stage.

Eventually, in the next step, two of the authors (ARS and AAK) will review the full text of the studies in order to determine the final studies, independently.

Any discordance in these steps will be resolved by consensus, and if the disagreement is not resolved, the opinion of a third expert (GS) will be used to resolve the case. 


\section{Risk of bias assessment in included studies}

We will use the Newcastle Ottawa Scale (NOS) for observational studies (for cross-sectional, retrospective and cohort studies) to assess the quality of final included studies. This scale has eight items including parts of selection, comparability and outcome (16).

To investigate the likelihood of a relationship between the quality of the preliminary studies and their results, the methodological quality of the included studies will be independently assessed by three independent authors (ARS, AK and HA). Any inconsistencies will be resolved by consensus, and if no agreement is reached yet again, the case will be resolved by seeking the views of a third expert (GS).

\section{Data extraction}

For final included studies, three authors (ARS and GS and HA) will independently extract the following summary data: first author name, study design, country, sample size, demographic variables of the participants, and duration of disease before liver transplantation, follow-up duration after the operation, cytomegalovirus infection, steroid consumption, smoking status, frequency (\%) of patients with improvement and frequency (\%) of patients with worsening of the course of disease, and use of tacrolimus or cyclosporine medication.

We will provide a summary of data in a table. In the absence of the required statistical data in the original studies, the authors will attempt to contact with their authors to obtain the appropriate data. The study will be eliminated if the author fails to respond to us for three times.

\section{Strategy for data synthesis}

The clinical course of the IBD would be evaluated both before and after liver transplantation. Accordingly, the frequency (\%) of patients with quiescent disease activity, improvement, or aggravation in disease severity would be assessed based on the data of studies. A battery of scoring systems have been applied in studies to assess the clinical course of IBD including Mayo score (disease activity index), simple clinical colitis activity index, need for hospitalization, need for escalation in medical therapy and Crohn's disease activity index, specifically for those with $\operatorname{CD}(3,10,12)$. Considering the lack of mean value and standard deviation of the scores in all relevant studies, and diversity of use of different score base instruments, we would include the frequency (\%) of the patients in remission or exacerbation after LT.

The result of the final original studies will be appeared concisely in a table encompassing the first author's name, year of publication, study design and the sample size and demographic data of the participants.

\section{Statistical analysis}

The pooled frequency (\%) for improved and exacerbated patients after transplantation, as a key measure, will be calculated in this study. The combination method will be based on methodological similarities in 
the included studies by the Fixed Effect Model or the Random Effect Model. If meta-analysis is not possible due to sever methodological heterogeneity, a narrative qualitative discussion based on findings from individual studies will be presented. Forest plots will be plotted for all the studies to show the separated and pooled frequency and their corresponding $95 \% \mathrm{Cls}$. The software used in the present study will be Stata V.14.1 (Stata Corp, college station, TX, USA).

\section{Assessment of heterogeneity}

The Q-statistic test and $\mathrm{I}^{2}$ statistics and their corresponding $95 \%$ Cls will be used to assess the statistical heterogeneity of the frequency (\%) values in the included studies. The following references according to Cochrane Handbook will be used as the bases for determining the degree of heterogeneity. Heterogeneity values of $0 \%-40 \%$ will be taken as 'perhaps not important'; Heterogeneity values of $30 \%-60 \%$ as 'moderate heterogeneity'; Heterogeneity values of $50 \%-90 \%$ as "substantial heterogeneity" and Heterogeneity values of $75 \%-100 \%$ as 'considerable heterogeneity' (17). The level of statistical significance will be set at $p<0.05$ for the Q-test.

\section{Sub group analysis}

Sub group analysis or meta-regression, if sufficient data are available, will be used appropriately to investigate the effect of statistical heterogeneity. In this study, variables such as age, sex, smoking, type of medication, and severity of IBD before transplantation are the variables that will be used in sub group analysis.

\section{Sensitivity analysis}

The one-out remove method will also be used for sensitivity analysis. If one of the combinations (K-1) of the studies shows a different result to the others, we will carefully consider the features of that study.

\section{Quality analysis}

Quality analysis will be performed if there is a statistically significant difference between the results of high-quality and low-quality studies.

\section{Assessment of publication bias}

If there are sufficient studies of more than 10, both Funnel Plot and Begg's and Egger's statistical tests will be used to evaluate publication bias. If the previously mentioned methods show evidence of the bias, the Fill \& Trim method will be used to correct the publication bias effect.

\section{Patient and public involvement}

No patients will be involved in this study

\section{Discussion}


This systematic review and meta-analysis study will clarify the correlation of IBD clinical course with liver transplantation. Because of the fact that all-inclusive and thorough methods in all the steps of this systematic review and meta-analysis will be used, the information gained from this study will be absolutely reliable.

Some of the most important restrictions of the future systematic review and meta-analysis are inconsistency of estimating the effect sizes in primary studies, lacking of a large multicenter study, and multiple bias level in the final included studies.

Because of the importance of inflammatory bowel disease, if the future study reveals consistent results, it will be clinically beneficial for physicians and other health care professionals to better manage inflammatory bowel disease after liver transplantation.

\section{Abbreviations}

IBD: Inflammatory Bowel Disease; PSC: Primary Sclerosing Cholangitis; UC: Ulcerative Colitis; Solid Organ Transplantation (SOT); LT: Liver Transplantation; CD: Crohn's disease; CMV: Cytomegalovirus; NOS: Newcastle Ottawa Scale.

\section{Declarations}

\section{Ethics approval and consent to participate}

Not applicable

Consent for publication

Not applicable

\section{Availability of data and materials}

Not applicable

\section{Competing interests}

The authors declare that they have no competing interests

\section{Funding}

This research received no specific grant from any funding agency in the public, commercial or not-forprofit sectors.

\section{Authors' contributions}


ARS is the guarantor of this study. All the authors contributed to the conception and design of the protocol as follows. ARS worked on the topic refinement, formulation of research question, review design, study selection forms, data extraction sheets and plan of analysis and wrote the protocol; ARS also designed the search strategy under the supervision of AAK, GRS, and MM. GRS, MM, HA and AAK contributed to the topic refinement, formulation of research question, review design and plan of analysis and gave critical feedback on the intellectual content of the draft protocol. GRS, AAK, ARS, HA and MM reviewed the manuscript for feedback. MM will review the articles and take care of the data extraction step along with ARS. AAK, MM, HA and GRS will take care of database management and carry out the literature search/handle the bibliography. As the senior author, GRS supervised the preparation of the study protocol and addressed the reviewers' comments. All the authors read and approved the final manuscript.

\section{Acknowledgements}

Hereby, the authors wish to express our gratitude to Professor Yunes Ghasemi, the Vice Chancellor of Research at Shiraz University of Medical Sciences for his specific support from systematic review and meta-analysis projects.

\section{References}

1. Steenstraten IC, Sebib Korkmaz K, Trivedi PJ, Inderson A, van Hoek B, Rodriguez Girondo MD, et al. Systematic review with meta-analysis: risk factors for recurrent primary sclerosing cholangitis after liver transplantation. Alimentary pharmacology \& therapeutics. 2019;49(6):636-43.

2. Palmela C, Peerani F, Castaneda D, Torres J, Itzkowitz SH. Inflammatory bowel disease and primary sclerosing cholangitis: a review of the phenotype and associated specific features. Gut and liver. 2018;12(1):17.

3. Fattahi MR, Malek-Hosseini SA, Sivandzadeh GR, Safarpour AR, Bagheri Lankarani K, Taghavi AR, et al. Clinical course of ulcerative colitis after liver transplantation in patients with concomitant primary sclerosing cholangitis and ulcerative colitis. Inflammatory bowel diseases. 2017;23(7):1160-7.

4. Navaneethan U, Venkatesh PG, Jegadeesan R, Lourdusamy V, Hammel JP, Kiran RP, et al. Comparison of outcomes for patients with primary sclerosing cholangitis associated with ulcerative colitis and Crohn's disease. Gastroenterology report. 2016;4(1):43-9.

5. Hosseini SV, Jafari P, Taghavi SA, Safarpour AR, Rezaianzadeh A, Moini M, et al. Fecal calprotectin is an accurate tool and correlated to seo index in prediction of relapse in iranian patients with ulcerative colitis. Iranian red crescent medical journal. 2015;17(2).

6. Bowlus CL, Lim JK, Lindor KD. AGA Clinical practice update on surveillance for hepatobiliary cancers in patients with primary sclerosing cholangitis: expert review. Clinical Gastroenterology and Hepatology. 2019;17(12):2416-22.

7. Tabibian JH, Bowlus CL. Primary sclerosing cholangitis: A review and update. Liver research. 2017;1(4):221-30. 
8. Schnitzler F, Friedrich M, Stallhofer J, Schönermarck U, Fischereder M, Habicht A, et al. Solid organ transplantation in patients with inflammatory bowel diseases (IBD): analysis of transplantation outcome and IBD activity in a large single center cohort. PLoS One. 2015;10(8).

9. Nannegari V, Roque S, Rubin DT, Quera R. A review of inflammatory bowel disease in the setting of liver transplantation. Gastroenterology \& hepatology. 2014;10(10):626.

10. Papatheodoridis G, Hamilton M, Mistry P, Davidson B, Rolles K, Burroughs A. Ulcerative colitis has an aggressive course after orthotopic liver transplantation for primary sclerosing cholangitis. Gut. 1998;43(5):639-44.

11. Gelley F, Miheller P, Péter A, Telkes G, Nemes B, editors. Activity of ulcerative colitis before and after liver transplantation in primary sclerosing cholangitis: the Hungarian experience. Transplantation proceedings; 2012: Elsevier.

12. Navaneethan U, Choudhary M, Venkatesh P, Lashner B, Remzi F, Shen B, et al. The effects of liver transplantation on the clinical course of colitis in ulcerative colitis patients with primary sclerosing cholangitis. Alimentary pharmacology \& therapeutics. 2012;35(9):1054-63.

13. Jørgensen KK, Lindström L, Cvancarova M, Karlsen TH, Castedal M, Friman S, et al. Immunosuppression after liver transplantation for primary sclerosing cholangitis influences activity of inflammatory bowel disease. Clinical Gastroenterology and Hepatology. 2013;11(5):517-23.

14. Haagsma E, Van Den Berg A, Kleibeuker J, Slooff M, Dijkstra G. Inflammatory bowel disease after liver transplantation: the effect of different immunosuppressive regimens. Alimentary pharmacology \& therapeutics. 2003;18(1):33-44.

15. Moher D, Shamseer L, Clarke M, Ghersi D, Liberati A, Petticrew M, et al. Preferred reporting items for systematic review and meta-analysis protocols (PRISMA-P) 2015 statement. Systematic reviews. 2015;4(1):1.

16. Stang A. Critical evaluation of the Newcastle-Ottawa scale for the assessment of the quality of nonrandomized studies in meta-analyses. European journal of epidemiology. 2010;25(9):603-5.

17. Higgins JP, Thomas J, Chandler J, Cumpston M, Li T, Page MJ, et al. Cochrane handbook for systematic reviews of interventions: John Wiley \& Sons; 2019.

\section{Supplementary Files}

This is a list of supplementary files associated with this preprint. Click to download.

- PRISMAPchecklist11.docx 